\title{
AVALIAÇÃO DOS ÍNDICES ZOOTÉCNICOS DE POEDEIRAS EM SISTEMA DE PRODUÇÃO CAGE-FREE
}

\author{
Evaluation of the zootechnical indexes of laying hens in a cage-free \\ production system
}

Daniela Cechin ${ }^{1 *}$; Carla Patricia Freitas²; Luciana Ruschel dos Santos²; Antônio Sergio do Amaral ${ }^{1}$; Daniela dos Santos de Oliveira ${ }^{1}$; Amito José Teixeira ${ }^{1}$

${ }^{1}$ Universidade Regional Integrada do Alto Uruguai e das Missões, URI Erechim, RS. *E-mail: dani. mv@bol.com.br

${ }^{2}$ Universidade de Passo Fundo, UPF - Passo Fundo, RS.

Data do recebimento: 01/03/2021 - Data do aceite: 13/08/2021

RESUMO: A mudança no padrão alimentar dos consumidores por produtos mais saudáveis e a preocupação com o bem-estar dos animais, levou o setor avícola a buscar alternativas para produção de ovos sem a utilização de gaiolas, como o sistema de produção cage-free. No entanto, poucos estudos investigaram a influência desse sistema nos índices zootécnicos da produção de ovos, o que levou ao desenvolvimento desse estudo de caso. No estudo foram utilizados 400 aves poedeiras da linhagem Isa Brown, com idade inicial de 12 semanas e os dados coletados na $17^{\mathrm{a}}$ semana de idade até $62^{\mathrm{a}}$ semana de idade. As variáveis analisadas foram o início da postura, pico de produção, produção de ovos, consumo de ração e a mortalidade das aves. Os resultados mostraram que as aves iniciaram a postura precocemente $\left(17^{\mathrm{a}}\right.$ semana de idade $)$ com pico de produção na $24^{\mathrm{a}}$ semana de idade e produtividade de 390 ovos/ dia. O consumo de ração foi de $1,661 \mathrm{~kg}$ para a produção de uma dúzia de ovos e mortalidade de $3 \%$ do lote. Com isso, demonstra que o sistema cage-free, em estudo, foi viável nos resultados dos índices zootécnicos, podendo, também, ser promissor quanto à nova exigência do mercado consumidor em relação ao bem-estar animal.

Palavras-chave: Avícola. Bem-estar animal. Gaiolas. Granja. Ovos. 
ABSTRACT: The change in the consumers' food pattern for healthier products and the concern with animal welfare has led the poultry sector to seek alternatives for egg production without the use of cages, such as the cage-free production system. However, few studies have investigated the influence of this system on the zootechnical indexes of egg production, which led to the development of this case study. The study used 400 laying hens of the Isa Brown lineage with an initial age of 12 weeks old and the data was collected from the 17 th week of age to 62 nd week of age. The variables analyzed were the beginning of laying, peak production, egg production, feed intake and bird mortality. The results showed that the birds started laying early (17th week of age) with peak production in the 24th week of age and productivity of 390 eggs/day. Feed consumption was $1,661 \mathrm{~kg}$ for the production of a dozen eggs and mortality of $3 \%$ of the flock. Thus, it demonstrates that the under study cage-free system was viable in the results of zootechnical indexes, and the new demand of the consumer market in relation to animal welfare may also be promising.

Keywords: Poultry. Animal welfare. Cages. Farm. Eggs.

\section{Introdução}

O setor avícola vem se destacando na produção de carnes e ovos, com avanços na criação devido ao uso de tecnologias, melhoramento genético e nutrição. Em 2020 o Brasil produziu mais de 53 bilhões de ovos/ ano e exportou um volume de 6.250 toneladas de ovos, sendo que $99,69 \%$ foram destinados ao mercado interno e $0,31 \%$ para exportação. Neste período foram alojadas $1.441 .548 \mathrm{mi}$ lhões/cabeças/ano de matrizes de postura e 124.317.339 milhões/cabeças de poedeiras comerciais/ano (ABPA, 2021).

A avicultura de postura intensificou sua produção com sistemas de criação em gaiolas, que consiste na automatização do fornecimento de alimento, distribuição de água, coleta dos ovos e excretas com o objetivo de maximizar o lucro e a produtividade com galinhas criadas em uma pequena área e maior produção de ovos (JONES et al., 2014). No entanto, esse sistema prejudicou o bem-estar das aves, reprimindo a expressão de seu comportamento natural. Na década de 1960 , na Europa, o bem-estar ganhou importância. Também, a mudança no padrão alimentar do consumidor por produtos mais saudáveis e seguros bem como a preocupação com o bem-estar dos animais, levou o setor avícola a buscar alternativas para produção de ovos sem a utilização de gaiolas. Apesar dos altos valores de produção, na avicultura de hoje, se torna obrigatório manter o bem-estar das galinhas. Com isso, vários estudos estão sendo realizados sobre as novas tendências de habitação para o bem-estar e a produção.

Os sistemas de produção sem gaiolas, ou seja, alternativos, proporcionam para as aves um ambiente com maiores espaços habitáveis e enriquecimento do bem-estar com uso de poleiros, ninhos e chão com cama, maravalha (HARTCHER; JONES, 2017). Dentre os sistemas alternativos existem dois modelos de galpões: os cage-free e o free-range.

Segundo a Humane Farm Animal Care (2018) estabeleceu as diretrizes da produção de ovos nesses sistema, para a obtenção de certificação da propriedade, sendo elas: os cage-free devem possuir uma densidade 
de alojamento com o mínimo de $0,14 \mathrm{~m}^{2}$ por poedeira, permitindo o comportamento natural das mesmas. No interior do galpão deve ser constituído por poleiros com 15 cm/ave, ninhos com proporção não inferior a um ninho para cada cinco aves, linhas de bebedouros e comedouros com fácil disponibilidade do alimento, com mínimo de $5 \mathrm{~cm}$ de espaço linear (para comedouros com acesso em ambos os lados) e cama/substrato de 250 $\mathrm{cm}^{2} /$ ave de material e tamanho de partícula adequado, de boa qualidade e ser manejada para permanecer em condição seca. As aves não tem acesso à área externa do galpão, mas possuem livre acesso a parte interna, podendo caminhar, ciscar, tomar banho de poeira, abrir e esticar as asas e realizam a postura dos ovos em ninhos.

No modelo free-range, as aves têm acesso livre à área externa do galpão durante a maior parte do dia, podendo assim tomar sol e/ou pastar. A área externa deve conter terra coberta por vegetação projetada, manejada e atualizada anualmente, minimizando o risco da área se tornar degradada, como também, distribuição apropriada de abrigos/sombras naturais, artificiais e coberturas. A quantidade mínima de área externa descoberta exigida é de $0,19 \mathrm{~m}^{2}$ por ave. As aves devem ter acesso à área externa por, no mínimo, seis horas diárias durante o período do dia e a distância do perímetro da cerca até a porta de um alojamento deve ser mais próximo de $366 \mathrm{~m}$. As aves devem ter áreas de saída suficientes: ter pelo menos $46 \mathrm{~cm}$ de altura e $53 \mathrm{~cm}$ de largura distribuídas em torno do alojamento, com, pelo menos, uma saída a cada 15 m na lateral do galpão, para garantir que todas as aves tenham acesso livre à área externa e permitir a passagem de mais de uma ave por vez.

Segundo Englmaierová et al. (2014) o sistema de criação utilizado, a temperatura, umidade relativa e a frequência de coleta dos ovos são fatores que influenciam na qualidade do produto. Já Alves et al. (2007) relatam que o sistema de criação em cama, quando devidamente projetado, pode ser compatível com o sistema de criação em gaiolas, possibilitando a obtenção do mesmo desempenho produtivo e qualidade de ovos produzidos. Mas, com a crescente conscientização da população mundial em relação ao bem estar animal e a disposição em pagar mais pelo produto livre de gaiola, o objetivo do estudo foi avaliar os índices zootécnicos de poedeiras mantidas no sistema de produção alternativo cage-free.

\section{Material e métodos}

A metodologia desenvolvida foi estudo de caso, que consiste no estudo profundo e exaustivo de um ou poucos objetos, de maneira que permita seu amplo e detalhado conhecimento (GIL, 2002).

$\mathrm{O}$ estudo de caso foi realizado em uma granja de aves de postura localizada no município de Jacutinga, norte do estado do Rio Grande do Sul, no período entre abril de 2019 e fevereiro de 2020, em um galpão de alvenaria, modelo tipo cage-free, com dimensões de $120 \mathrm{~m}^{2}$, sendo $4 \mathrm{~m}^{2}$ de largura e $30 \mathrm{~m}^{2}$ de comprimento, e dividido em dois compartimentos para dois lotes com capacidade de 6,66 aves por metro quadrado. $\mathrm{O}$ galpão era cercado por lonas com sistema de catracas para abertura e fechamento das lonas. Os comedouros eram do tipo tubular, bebedouros pendulares sendo eles intercalados e posicionados no centro, ocupando toda extensão do galpão. Nas laterais estavam inseridos os poleiros feitos em madeira e do outro lado os ninhos, sendo estes com capacidade para cinco aves/ninho, construídos de madeira e no seu interior foram adicionados uma camada de $10 \mathrm{~cm}$ de maravalha de pinos. O chão do galpão era coberto por $250 \mathrm{~cm}^{2} /$ ave de maravalha de pinus (figura 1). 
Foram utilizadas 400 poedeiras da linhagem Isa Brown, com 12 semanas de idade. As aves foram alimentadas com dietas basais, isocalóricas e isoproteicas, produzidas na própria granja, de acordo com a recomendação das Tabelas Brasileiras para Aves e Suínos (ROSTAGNO et al., 2017), formuladas por fase de vida: inicial $\left(12^{\mathrm{a}}\right.$ semanas até $15^{\mathrm{a}}$ semanas), pré-postura (até início da produção - $17^{\mathrm{a}}$ semanas), postura ( $17^{\mathrm{a}}$ semana até $62^{\mathrm{a}}$ semanas). As dietas foram fornecidas duas vezes ao dia, no período da manhã e tarde. A água fornecida era de poço artesiano, clorada e anualmente avaliada quanto aos parâmetros físico-químicos e microbiológicos.

Figura 1 - Galpão sistema Cage-free e equipamentos

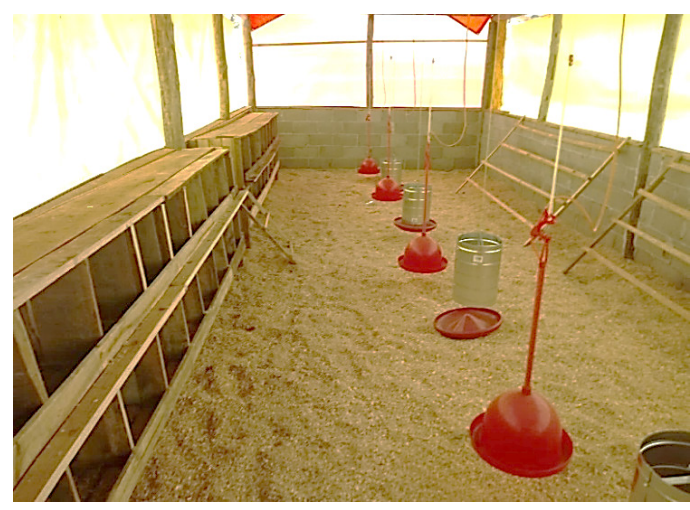

A temperatura foi controlada através de termômetros com a abertura (dias mais quentes) e fechamento (dias frios) de cortinas, manualmente. A parte externa do galpão possui uma barreira natural de árvores para proteção frente a correntes de ventos e manutenção de microclima ameno.

O programa de luz foi realizado com luz artificial das $20 \mathrm{~h}$ às $22 \mathrm{~h}$ e das $03 \mathrm{~h}$ às $07 \mathrm{~h}$, com intervalo de 5 horas de escuro e luz natural durante o restante do dia. $\mathrm{O}$ fornecimento de horas luz (artificial) estimula as aves à postura dentro dos ninhos, pois sem luz as aves iniciam a postura de madrugada no chão do aviário, causando um maior descarte de ovos por contaminação e trincas. Os ovos foram coletados manualmente, duas vezes ao dia, as $07 \mathrm{~h} 30 \mathrm{~min}$ e $15 \mathrm{~h}$.

As variáveis estudadas foram: início de postura (semanas), pico de produção (semanas) produção de ovos (unidade), consumo de ração $(\mathrm{g})$ e mortalidade de aves (\%) em um período de $62^{\mathrm{a}}$ semana, com coleta diária de dados iniciados na $17^{\mathrm{a}}$ semana de idade.

\section{Resultados e Discussão}

As aves desse estudo iniciaram a postura na $17^{\mathrm{a}}$ semana de vida, o que difere do estudo realizado por Santos et al. (2011), em que as aves iniciaram a postura por volta da $20^{\mathrm{a}}$ semana de idade. Essa precocidade na postura dos ovos está associada ao melhoramento genético e à dieta balanceada no momento da cria e recria (SANTOS, 2020). No entanto, durante as três primeiras semanas, as aves produziram ovos de tamanhos inferiores do padrão comercial, representando $35,55 \%$ dos ovos produzidos no primeiro mês da postura, só alcançando o tamanho padrão na $20^{\mathrm{a}}$ semana de vida.

Alguns estudos relatam que os diferentes sistemas de produção acomete a massa dos ovos, além de existir uma correlação positiva entre o peso dos ovos e o peso corporal das aves (ONBAŞILAR et al., 2015; ZHANG et al., 2005).

A Figura 2 apresenta os resultados da produção de ovos (unidade) conforme a idade da ave (semanas).

No entanto, os dados apresentados diferem de um estudo realizado por Santos et al. (2011), com pico de produção na $29^{\mathrm{a}}$ e $30^{\mathrm{a}}$ semana. Ainda, Duarte (2016) encontrou resultados na $20^{\mathrm{a}}$ semana de idade, com $5 \%$ do lote iniciando a fase de postura com pico de produção entre a $26^{\mathrm{a}}$ e $30^{\mathrm{a}}$ semana. Porém, 
nessa fase de idade, a produção começa a decrescer, sendo essas alterações ocasionadas, principalmente, pelo período de luz utilizado nas fases de cria e recria (SANTOS et al., 2011).

Figura 2 - Produção de ovos conforme a idade da ave de postura

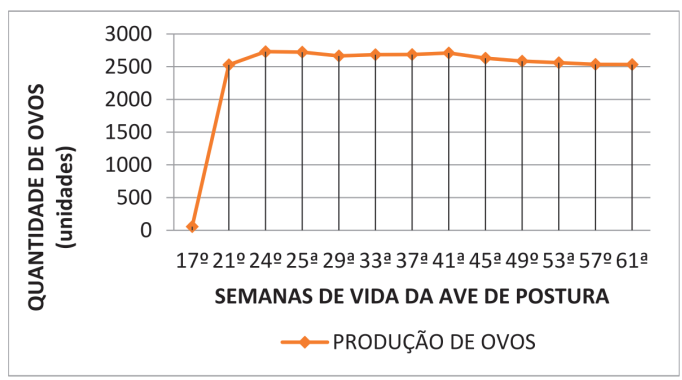

Após o início da postura ( $17^{\mathrm{a}}$ semana), houve um aumento considerável na $21^{\text {a }}$ semana, atingindo o pico de produção na $24^{\mathrm{a}}$ semana com produção de 390 ovos/dia, mantendo-se o pico até a $41^{\mathrm{a}}$ semana e um declínio gradual até a $61^{\mathrm{a}}$ semana. A quanti- dade de ovos produzidos diminui conforme aumenta a idade das aves, entre 18 e 20 meses de idade, quando ocorre a muda de penas e a produção de ovos cessa (EMBRAPA, 2004).

A Tabela I mostra os resultados do consumo de ração (g/ave/dia) conforme a idade da ave e produção de ovos/dia em unidade.

As aves iniciaram a postura recebendo $87 \mathrm{~g} /$ dia de ração na $17^{\mathrm{a}}$ semana e ajustada para 120 a $125 \mathrm{~g} /$ dia de ração na $21^{\mathrm{a}}$ semana. Conforme Santos (2020), esta fase inicial de postura deve ser acompanhada de consumo de água e ração para que haja boa uniformidade e desenvolvimento de carcaça da ave.

No pico de produção de ovos, ou seja, na $24^{\mathrm{a}}$ semana, a quantidade consumida foi de $135 \mathrm{~g} / \mathrm{dia} / \mathrm{ave}$. Após o pico, essa quantidade foi readequada, mantendo em $128 \mathrm{~g} / \mathrm{dia} / \mathrm{ave}$. O consumo de ração depende do tamanho da ave, da velocidade de produção de ovos, da temperatura nos galpões e do nível energético da alimentação, sendo geralmente necessários dois quilos de ração para a produção de uma dúzia de ovos (EMBRAPA, 2004).

Tabela I - Produção de ovos e consumo de ração conforme a semana de vida das aves postura

\begin{tabular}{ccc}
\hline Semana de vida das aves & Consumo de ração (g/ave/dia) & $\begin{array}{c}\text { Produção de ovos/dia } \\
\text { (unidade) }\end{array}$ \\
\hline 17 & 87 & 8 \\
21 & 125 & 361 \\
24 & 135 & 390 \\
25 & 137 & 389 \\
29 & 132 & 380 \\
33 & 128 & 383 \\
37 & 128 & 384 \\
41 & 127 & 387 \\
45 & 128 & 375 \\
49 & 128 & 369 \\
53 & 128 & 366 \\
57 & 128 & 362 \\
61 & 128 & 362 \\
\hline
\end{tabular}


Estudos realizados com diferentes linhagens de poedeiras mostraram que em sistema caipira tiveram uma conversão alimentar mais pobre em comparação com baias de gaiolas e aviários. Ainda, a comparação de sistemas de gaiolas convencionais e enriquecidos teve um efeito significativo nos resultados de conversão alimentar (ENGLMAIEROVA et al., 2014; ONBAŞILAR et al., 2015). Também, Ahammed et al. (2014) observaram maior consumo de ração e taxa de conversão no sistema de criação em gaiolas e aviário.

No pico de produção de ovos (24 $4^{\mathrm{a}}$ semana) foram necessários $1,661 \mathrm{~kg}$ de ração para a produção de uma dúzia de ovos, enquanto na $42^{\mathrm{a}}$ semana utilizou-se $1,668 \mathrm{~kg}$ de ração para produção de uma dúzia de ovos.

A quantidade de ração deve ser aumentada ou reduzida de acordo com o estado corporal, lembrando que as futuras poedeiras devem estar bem nutridas, sem obesidade (EMBRAPA, 2004).

A mortalidade das aves poedeiras é outro fator importante a ser estudado. Barboza (2019) divide em seis categorias as principais causas de mortes: natural, caracterizada por encontrar o animal sem nenhuma lesão ou indicativo de morte específica; súbita, parte do corpo com a coloração roxa e em posição de decúbito dorsal; por canibalismo, sacrificadas em função de lesões ocasionadas ao canibalismo; por prolapso, sacrificadas em resultado da exposição do oviduto até a cloaca; por amontoamento, indicada através de uma grande quantidade de animais mortos após pânico.

No lote em que foi realizado o estudo houve a morte de 12 aves, sendo oito por esmagamento, já que as aves permaneciam boa parte do dia amontoadas em uma lateral do galpão Essas mortes foram ocasionadas a partir da $2^{\mathrm{a}}$ semana do alojamento, enquanto outras três aves foram sacrificadas por exposição de oviduto, nas $22^{\mathrm{a}}, 23^{\mathrm{a}}$ e $24^{\mathrm{a}}$ semana, período de pós-postura.

Um dos maiores problemas da criação de aves soltas ao chão (sistema cage-free) é o amontoamento das aves, que acontece devido ao costume de se amontoar sempre em um lado do aviário, o que pode causar mortes por sufocamento ou esmagamento. Em experimentos realizados por Barboza (2019) com três lotes de aves de postura, ambas em sistema livre de gaiolas, dois lotes apresentaram maior mortalidade na fase de pós-postura, sendo a maior causa amontoamento, seguida por morte natural.

No estudo conduzido, a mortalidade representou $3 \%$ no lote, considerando o período desde a chegada das aves na granja $\left(12^{\mathrm{a}}\right.$ semanas) até as $62^{\mathrm{a}}$ semanas de vida. Já em experimento conduzido por Jácome (2005), com poedeiras de $24^{\mathrm{a}}$ a $40^{\mathrm{a}}$ semanas, em diferentes sistemas de instalações, a mortalidade foi praticamente nula. Ainda, Filho (2004), em seu estudo comparando a mortalidade de aves soltas e de gaiolas, cita que ocorreu mortalidade somente no sistema de gaiolas e para condições de estresse térmico.

\section{Considerações Finais}

O sistema cage-free ainda é recente no Brasil e caminha em um ritmo lento, mas promissor pois permite que as aves expressem seus comportamentos naturais além da tendência de valorização de produtos que são cultivados levando em consideração os cuidados com o meio ambiente e com o bem-estar dos animais. Portanto, esse estudo contribuiu mostrando que a produção de ovos em sistema cage-free foi viável. 


\section{REFERÊNCIAS}

ABPA. Associação Brasileira de Proteína Animal. Relatório Anual 2021. Disponível em: http:// abpa-br.org/wp-content/uploads/2021/04/ABPA_Relatorio_Anual_2021_web.pdf. Acesso em: 10 mar. 2021.

AHAMMED, A.; CHAE, B. J.; LOHAKARE, J.; KEOHAVONG, B.; LEE, M. H.; LEE, S. J.; KIM, D. M; LEE, J. Y.; OHH, S.J. Comparison of aviary, barn and conventional cage raising of chickens on laying performance and egg quality. Asian-Austral. J. Animal, v. 27, p. 1196-1203, 2014. https:// www.animbiosci.org/upload/pdf/ajas-27-8-1196-17.pdf. Acesso em: 10 mar. 2021.

ALVES, P. S.; SILVA, O. J. I.; PIEDADE, S. M. S.; Avaliação do bem-estar de aves poedeiras comerciais: efeitos do sistema de criação e do ambiente bioclimático sobre o desempenho das aves e a qualidade de ovos. Revista Brasileira de Zootecnia, v. 26, n. 5, p. 1388-1394, 2007. Disponível em:https://www.researchgate.net/publication/237467287_Avaliacao_do_bemestar_de_ aves_poedeiras_comerciais_efeitos_do_sistema_de_criacao_e_do_ambiente_bioclimatico_sobre_o_ desempenho_das_aves_e_a_qualidade_de_ovos. Acesso em: 09 dez. 2019.

BARBOZA, C. F; Avaliação do índice de mortalidade de poedeiras comerciais no sistema livre de gaiolas em uma granja do município de Jaboticabal - SP. 2019. 26 f. Trabalho de Conclusão de Curso (Graduação em Zootecnia) - Universidade Federal de Uberlândia, Uberlândia, 2019. Disponível em: https://repositorio.ufu.br/bitstream/123456789/27924/3/Avalia\%C3\%A7\%C3\%A3o \%C3\%8DndiceMortalidade.pdf. Acesso em: 09 dez. 2019.

DUARTE, C. A. H. G. O efeito do peso vivo às 17 semanas de idade de galinhas poedeiras nos parâmetros produtivos e de qualidade do ovo durante a fase de postura. Dissertação de mestrado, Universidade de Lisboa, Lisboa, Portugal, 2016. Disponível em: https://www.repository. utl.pt/handle/10400.5/11642. Acesso em: 12 jun. 2020.

EMBRAPA INFORMAÇÃO TECNOLOGICA. Manual de Segurança e Qualidade para a Avicultura de Postura. Projeto Pas Campo, 2004. Disponível em: https://www.embrapa.br/buscade-publicacoes/-/publicacao/111866/manual-de-seguranca-e-qualidade-para-a-avicultura-de-postura. Acesso em: 09 dez. 2019.

ENGLMAIEROVA, M.; TUMOVA, E.; CHARVATOVA, V.; SKRIVAN, M. Effects of laying hens housing system on laying performance, egg quality characteristics, and egg microbial contamination. Czech Journal Animal Science, v. 59. p. 345-352, 2014. Disponível em: https:// www.agriculturejournals.cz/publicFiles/130012.pdf. Acesso em: 02 abr. 2021.

FILHO, B. D. A. J. Avaliação do bem estar de aves poedeiras em diferentes sistemas de produção e condições ambientais, utilizando análise de imagens. Dissertação de Mestrado, Escola Superior de Agricultura Luiz de Queiroz, Universidade de São Paulo, Piracicaba, 2004. Disponível em: https://teses.usp.br/teses/disponiveis/11/11131/tde-11052005-144156/publico/jose.pdf. Acesso em: 12 jan. 2020.

GIL, A. C. Como elaborar Projetos de Pesquisa. 4. ed. São Paulo: Atlas, 2002. Disponível em: http:// www.uece.br/nucleodelinguasitaperi/dmdocuments/gil_como_elaborar_projeto_de_pesquisa.pdf. Acesso em: 13 abr. 2021.

HARTCHER K.; JONES, B. The welfare of layer hens in cage and cage-free housing systems. World's Poultry Science Journal, v. 73 n. 4 p. 767-782, 2017. Disponível em: https:// www.tandfonline.com/doi/pdf/10.1017/S0043933917000812?needAccess=true. Acesso em: 10 mar. 2021. 
HUMANE FARM ANIMAL CARE. Galinhas Poedeiras: Referencial de Bem-Estar Animal. Certified Humane Brasil, 2018. Disponível em: http://certifiedhumane.org/wp-content/uploads/ HFAC-GalinhasPoedeiras18v2-1.pdf. Acesso em: 10 mar. 2021.

JÁCOME, D. T. M. I. Efeitos de dois sistemas de acondicionamento sobre os índices ambientais e zootécnicos de poedeiras semi pesadas. Dissertação de mestrado UFCG, Campina Grande: 2005. Disponível em: http://dspace.sti.ufcg.edu.br:8080/jspui/handle/riufcg/1077. Acesso em: 15 jan. 2020. JONES, D.R.; KARCHER, D.M.; ABDO. Z. Effect of a commercial housing system on egg quality during extended storage. Poultry Science, v. 93, p. 1282-1288, 2014. Disponível em: https://www. sciencedirect.com/science/article/pii/S0032579119361267?via\%3Dihub. Acesso em: 4 abr. 2021.

ONBASILAR, E. E.; ÜNAL, N.; ERDEM, E.; KOCAKAYA, A.; YARANOGLU, B. Production performance, use of nest box, and external appearance of two strains of laying hens kept in conventional and enriched cages. Poultry Science, v. 94, p. 559-564, 2015. Disponível em: https:// www.sciencedirect.com/science/article/pii/S003257911932437X. Acesso em: 5 abr. 2021.

ROSTAGNO, H. S.; ALBINO, L. F. T.; DONZELE, M. I. H. J. L.; SAKOMURA, N. K.; PERAZZO, F. G.; SARAIVA, A.; TEIXEIRA, M. L.; RODRIGUES, P. B.; OLIVEIRA, R. F.; BARRETO, S. L. 18 T.; BRITO, C. O. Tabelas brasileiras para aves e suínos: composição de alimentos e exigências nutricionais. Viçosa (MG): Editora UFV, 4 ed., p.488, 2017. Disponível em:https://edisciplinas.usp. br/pluginfile.php/4532766/mod_resource/content/1/Rostagno\%20et\%20al\%202017.pdf. Acesso em: 5 abr. 2021.

SANTOS, A. T.; GERALDO, A.; GOMES, R. A. K.; MOREIRA, N. J.; MACHADO, C. L.; RODRIGUES, M. L.; TELES, C. M.; OLIVEIRA, L. M.; FREITAS, S. L. Avaliação de desempenho de poedeiras Isa Brown criadas no IFMG - campus Bambuí em comparação aos dados de desempenho descritos no Manual da Linhagem. IV Jornada Científica, Minas Gerais, 2011 Disponível em: https://www.bambui.ifmg.edu.br/jornada_cientifica/2011/resumos/zootecnia/63.pdf. Acesso em: 15 ago. 2020.

SANTOS, R. P. M. Produção de poedeiras comerciais em fase de cria e recria. Monografia (Graduação em Zootecnia) - Universidade Federal de Sergipe, Núcleo de Graduação em Zootecnia, Nossa Senhora da Glória, 2020 Disponível em: https://ri.ufs.br/jspui/handle/riufs/13169. Acesso em: 12 jun. 2020.

ZHANG, L.C.; NING, Z. H.; XU, G. Y.; HOU, Z. C.; YANG, N. Heritabilities and genetic and phenotypic correlations of egg quality trans in Brown egg dwarf layers. Poultry Science, v. 84, p. 1209-1213, 2005. https://www.sciencedirect.com/science/article/pii/S0032579119446555. Acesso em: 8 mar. 2021. 